\title{
How Medicine Informs Informatics: Information is Asymmetry, Not Entropy $^{\S}$
}

\author{
Hector Sabelli
}

Chicago Center for Creative Development, 2400 N. Lakeview, Chicago, Illinois 60614, USA

\begin{abstract}
A concept of information as asymmetry in multiple dimensions is based on the demonstration that causal action, not hypothetical chance events, is a parsimonious explanation of novelty. The study of heartbeat variation revealed creative features in causal processes, namely greater novelty than random variation, decrease in entropy, temporal change in pattern, and progressive increase in variance. These properties characterize a new type of process, Bios. Bios has been demonstrated at all levels of organization from Number Theory and Quantum Physics to Population Dynamics and Economics. Mathematical experiments show that Bios is generated causally by generic processes present at multiple levels of organization: (1) unidirectional action, (2) bipolar opposition (e.g. electromagnetic energy, sex, mutual feedback) and (3) conservation. These studies underlie the formulation of information as $1,2,3, \ldots, \mathrm{N}$ dimensional order (i.e. asymmetry and transitivity). Thus information channels flux into flow, cycle, structure, and more complex organization in nature. Human reasoning and computer logic may be guided by this logic of nature.
\end{abstract}

Keywords: Bios, complexity, entropy, information, quantum physics, time series analysis.

\section{INTRODUCTION}

When the Europeans reached the New World, they brought their culture to the Native Americans, and in turn they took to Europe not only silver and potatoes, but also science and technology -e.g. Native Americans were much more advanced in pharmacology. In the same manner, the introduction of computers has brought many advances in medicine, but in turn medicine may bring new concepts into Informatics. Consider the widespread notions of information as entropy, entropy as disorder, randomness as complexity, and innovation as the result of independent chance events. This article presents alternatives to each of these notions. I apologize for the density of this article, covering major issues in few sentences, so understanding depends on consulting its references. This is unavoidable in the presentation of an integrative theory of information, its potential application to computation, and its human implications.

Medicine provides a new manner of thinking.

Good medicine is scientific, comprehensive and therapeutic. Scientific means seeking natural and rational explanations; computers foster them through the analysis and modeling of complex processes. Medicine and science were born as 'natural philosophy', as contrasted to supernatural myth. Human processes are natural processes; informatics is natural science. Medicine is by necessity comprehensive, as health and illness involve physical, social and psychological processes. Informatics is also multidisciplinary; it deals with natural and artificial systems, and has computational, cognitive and social aspects. But being cross-disciplinary is not

*Address correspondence to this author at the Chicago Center for Creative Development, 2400 N. Lakeview, Chicago, Illinois 60614, USA; E-mail: Hector_Sablli@rush.edu

$\S$ Title of an invited lecture to be delivered at the 6th International Conference on Informatics and Systems, Cairo, Egypt, 2008. enough. To be scientific, a science needs to integrate knowledge. Each discipline can and must learn from the others. What sounds scientific in a given discipline cannot be true if it contradicts what we know to be true in other sciences. Many economic 'theories' dissolve when their assumptions regarding rationality and self-interest are contrasted with psychological data. What is demonstrable false in one science cannot be adopted as an assumption in another.

Consideration of simple processes can guide us to understand complex ones, as illustrated by genetics. In turn genetics offer a model for the causal -not random-- generation of diversity, novelty and complexity from simple origins. Consideration of complex phenomena can guide us to also explore simple ones (complexity inferences [1]) as will be illustrated later.

Medicine is also therapeutic: Human sciences require a humane perspective, a choice of what is significant and desirable to study, not 'profitable'. Human science without a humanistic standpoint is a contradiction in terms. Considering the human implications of our assumptions, methods, and problems we choose to study fosters rather than weaken the scientific method.

\section{IDENTIFYING CREATIVE PROCESSES}

The starting point of this research is the study of heartbeat series that led to the concept of creative, life-like (biotic) processes. Their apparently regular sequence actually includes a small but vital variation; in fact cardiac regularity predisposes to cardiac failure and complete regularity predicts death within 24 hours [2]. Heartbeat variation reveals features not previously observed in natural or mathematical series, namely increases in variance [3], novelty [4,5] and complexity $[4,5]$ with time. These features are detected and quantified through the development of new methods for time series analysis [5] that focus on variation in time and dimension (revealed by embeddings) rather than on invariance, 
measuring complexity at a single embedding at one instant of time.

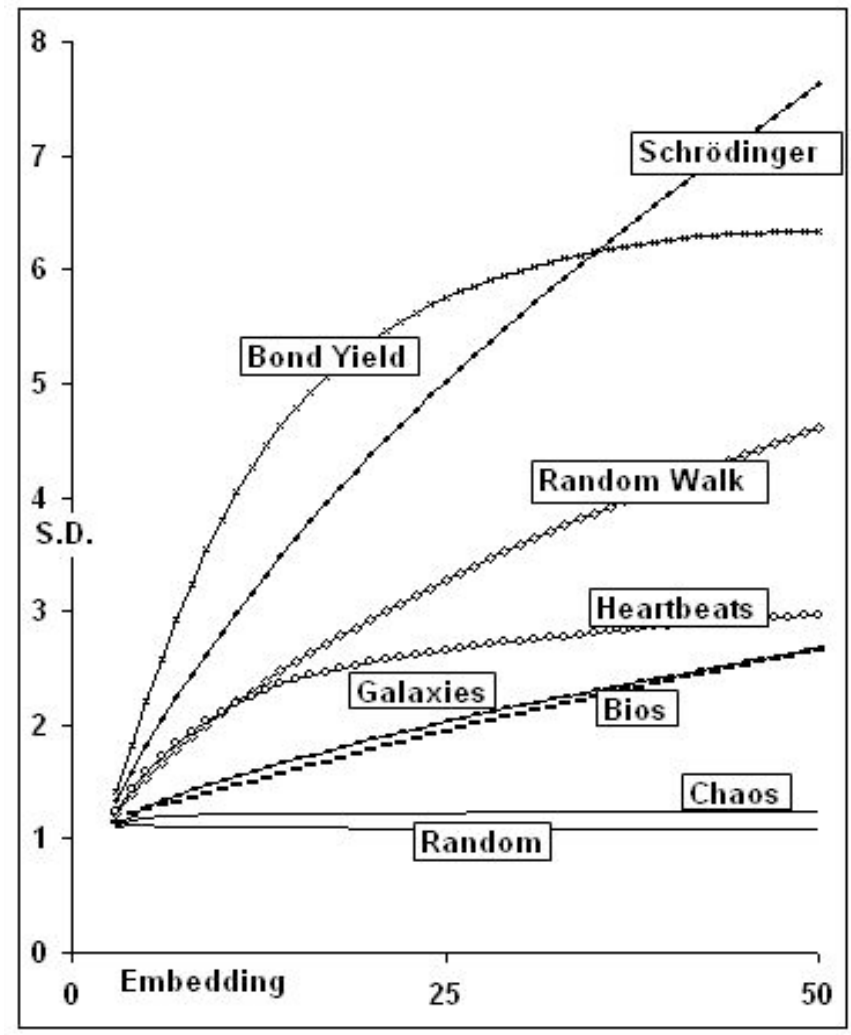

Fig. (1). Diversification: The standard deviation (SD) is computed for sets of $2,3, \ldots \mathrm{N}$ consecutive terms of the time series, starting with each term in the series; the values obtained for each embedding are averaged for the entire series, and these averages are plotted as a function of the number of embeddings.

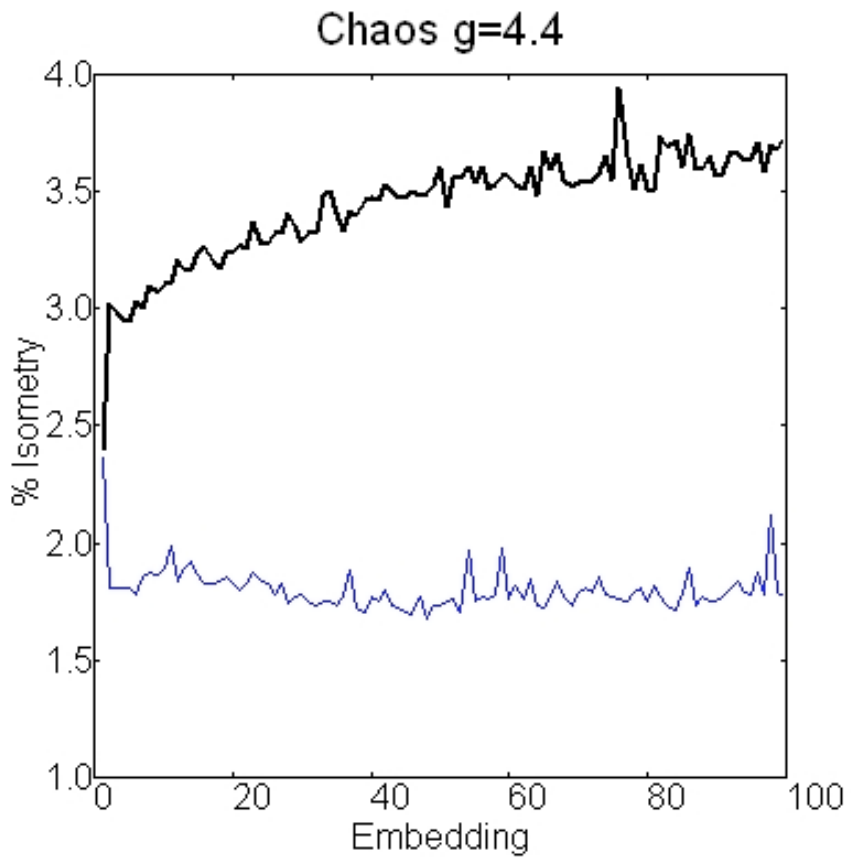

Diversification is the increase in variance with time characteristic of creative processes. It is most accurately detected by measuring standard deviation (SD) at increasing embedding dimensions [5]. The measurement of changes in variance as a function of time or dimensionality distinguishes three types of processes (Fig. 1). Random distributions are diverse (see section 6) but do not diversify; as mechanical processes, they maintain a stable variance after the first few embeddings. Other processes first converge to equilibrium, periodic or chaotic attractors, and from there on their variance remains stable. Creative natural processes show diversification, i.e. increase in S.D. with time (global diversification) or embedding (local diversification (Fig. 1). Finding diversification challenges the current focus of Dynamics on attractors, and highlights creative processes that diverge from them. Note: information increases with variance; standard statistics makes information inversely proportional to variance.

Novelty (Fig. 2) is the innovation generated spontaneously by creative processes. It is indicated by the low recurrence of isometric vectors, and measured as its increase by shuffling the data [6]. The Euclidean norms of vectors of $\mathrm{N}$ consecutive terms of the series are calculated starting with each term, and compared. When their difference is less than a given radius of tolerance, an isometry is counted. (Note: novelty is detected by isometry, not by other types of recurrence; a program is available at http \creativebios.com). Since a recurrence is a repetition of pattern, a lower than random recurrence rate indicates that the process under consideration innovates more than chance events. Novelty is a defining feature of Bios. Chaos has a large number of isometry recurrences (Fig. 3), even larger than its randomized copies (Fig. 2). Heartbeat series show novelty, hence variation (causal),

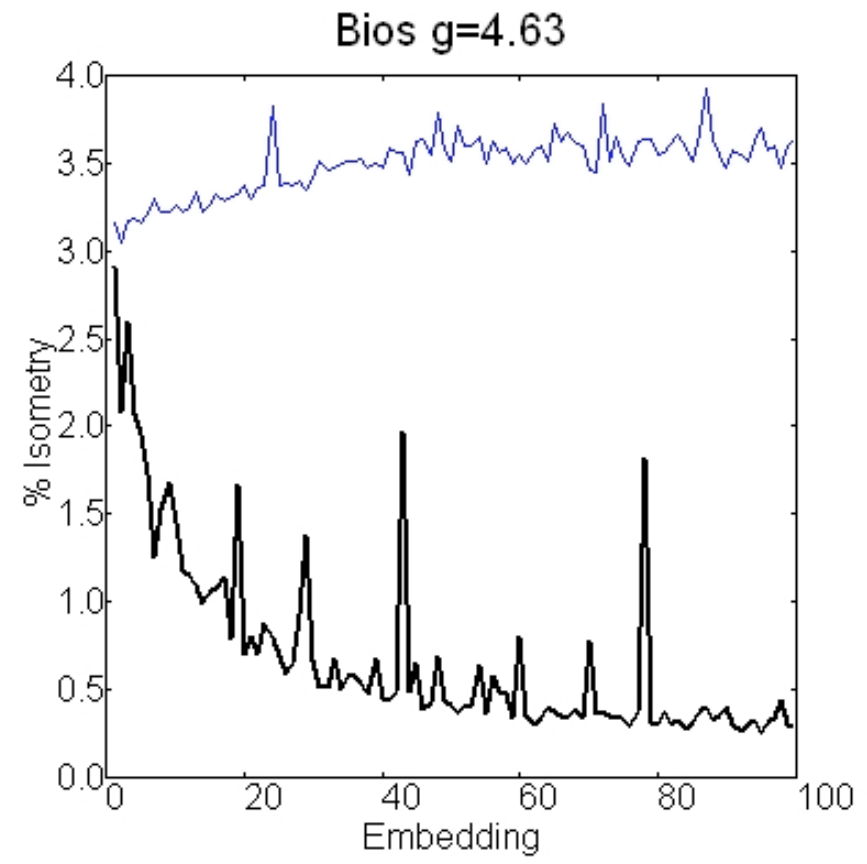

Fig. (2). Novelty: the percentage of isometries is plotted as a function of the embedding. Series generated by the process equation: $[A(t+1)=$ $\mathrm{A}(\mathrm{t})+\mathrm{g} * \sin (\mathrm{A}(\mathrm{t}))]$. Bios and Chaos and their shuffled copies (thin line). In Bios, the percentage of isometries decreases with embedding, in contrast to the increase in the shuffled copy. In Chaos, the number of isometries remains large and higher than in the shuffled copy. 
not variability (chance). The generation of unique individuals by reproductive processes is paradigmatic of causal novelty.

The demonstration that causal novelty is greater than chance variability challenges the assumption of 'chance' and randomness as parsimonious 'explanations' for change. Do not assume chance unless proven. We may or may not find at once the cause of a given process, but declaring it to be random positively prevents us from discovering it [7]. It stops seeking causality, and it encourages the destruction of undetected patterns, e.g. the destruction of nature by industrialism [7]. A scientist should not assume randomness unless demonstrated -an essential reversal of the notion that one should not assume causality unless proven, that has been dogmatically held as self-evident. Just as to dismiss a fact as mere chance and an observation as mere coincidence is bad medicine, it often is bad science.

The generation of complexity by simpler processes is the essential characteristic of creativity. Temporal complexity is detected by recurrence plots (Fig. 3) as well as by wavelet plots. While the pattern of random and chaotic series is uniform -the more they change, the more they stay the same--, truly creative processes generate qualitative changes in pattern. In recurrence plots, clusters of recurrences ('complexes') are separated by recurrence free intervals. Complexity is thus defined by qualitative changes in temporal pattern [8]. One can also demonstrate complexity in the frequency domain. Power is an inverse function of frequency : power is high for simple slow frequencies while complexity requires also higher frequencies. 1/f power spectrum is characteristic of many natural processes; it is found in biotic processes but not in chaotic series such as those generated by the logistic equation [9].

\section{BIOS}

Displaying diversification, novelty and complexification, characteristics which are absent in random, periodic, or chaotic series, heartbeats exemplify a previously unrecognized pattern, which has been dubbed Bios, meaning life [8].

Table 1 summarizes the similarities and differences between bios and other aperiodic processes. Both bios and chaos are causal but only bios displays the characteristics of creativity (diversification, novelty and temporal complexification) observed in natural processes. Just as complex random walks can be constructed by integrating random changes, complex Bios can be constructed by integrating chaos series with 0 mean. Bios and stochastic noise are creative, but only bios is causal. The series of differences between consecutive terms of biotic series display pattern, biotic in the case of heartbeat series and chaotic in biotic series generated by the process equation. Stochastic processes generated by the addition of random changes may also display features of creativity, but in these cases, change being random, the series of differences between consecutive terms do not show pattern.

As the biotic pattern had not been previously identified, one might have thought that it was uniquely found in heart-
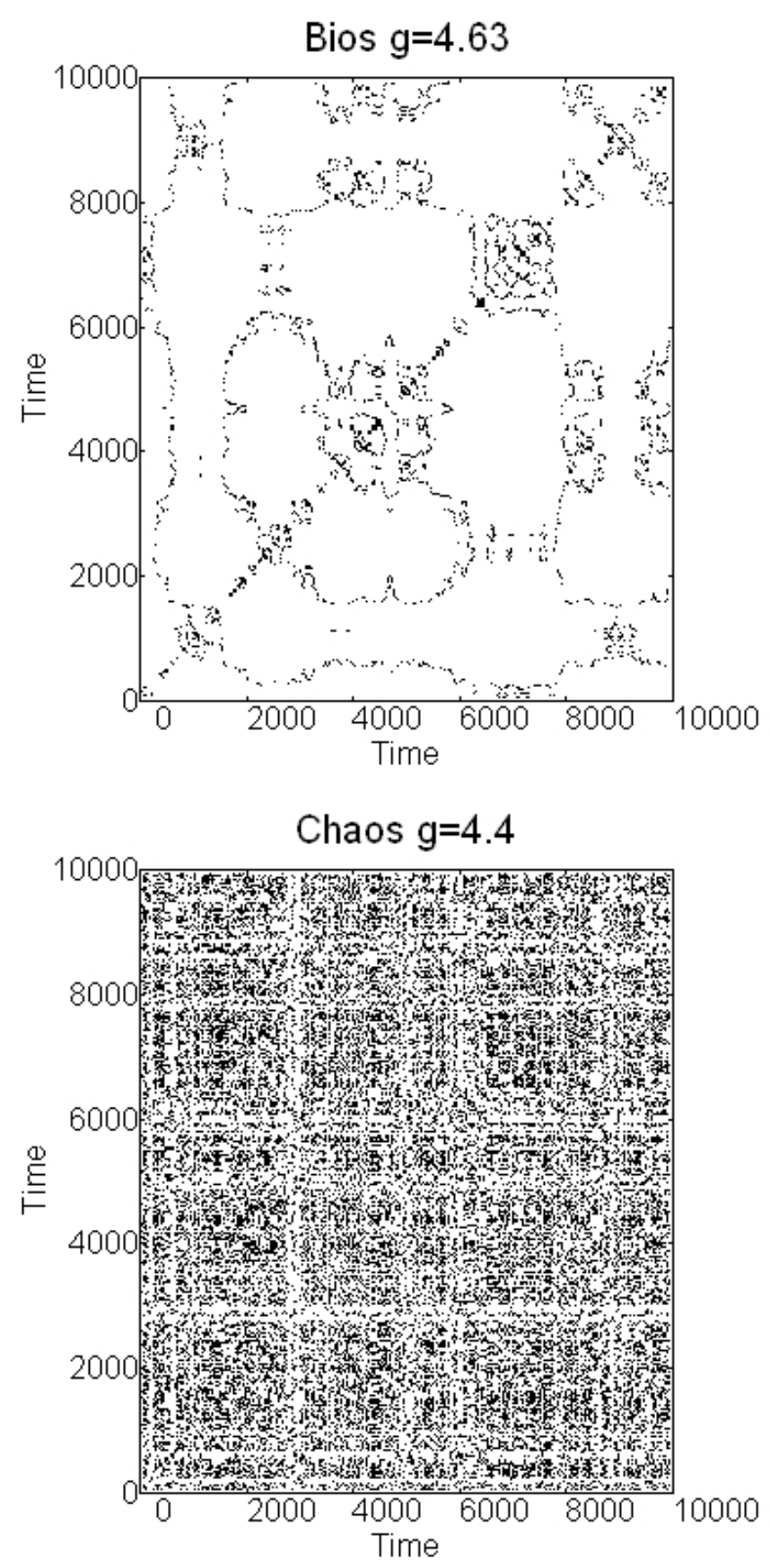

Fig. (3). Recurrence plots of series generated with the process equation. Top: Bios. Bottom: chaos. When the feedback gain is high, there are few isometries that are organized in clusters (complexes). Chaos: At lower gain, isometries are abundant and distributed uniformly.

beat series. On the contrary, biotic patterns are found at all levels of organization: (1) Mathematics: prime numbers [10] and the related Riemann equation [11]. (2) Quantum physics: Schrödinger wave function [12], and its relativistic version, the Klein-Gordon-Fock equation [13]. (3) Cosmology: temporal distribution of galaxies [12-14] and quasars [13], and gravitational waves [Sabelli et al., in preparation]. (4) Planetary: air and ocean temperature, river levels, river and shore fractal forms [8]. (5) Molecular biology: sequences of bases in DNA [8]. (8) Physiology: heartbeat series and respi- 


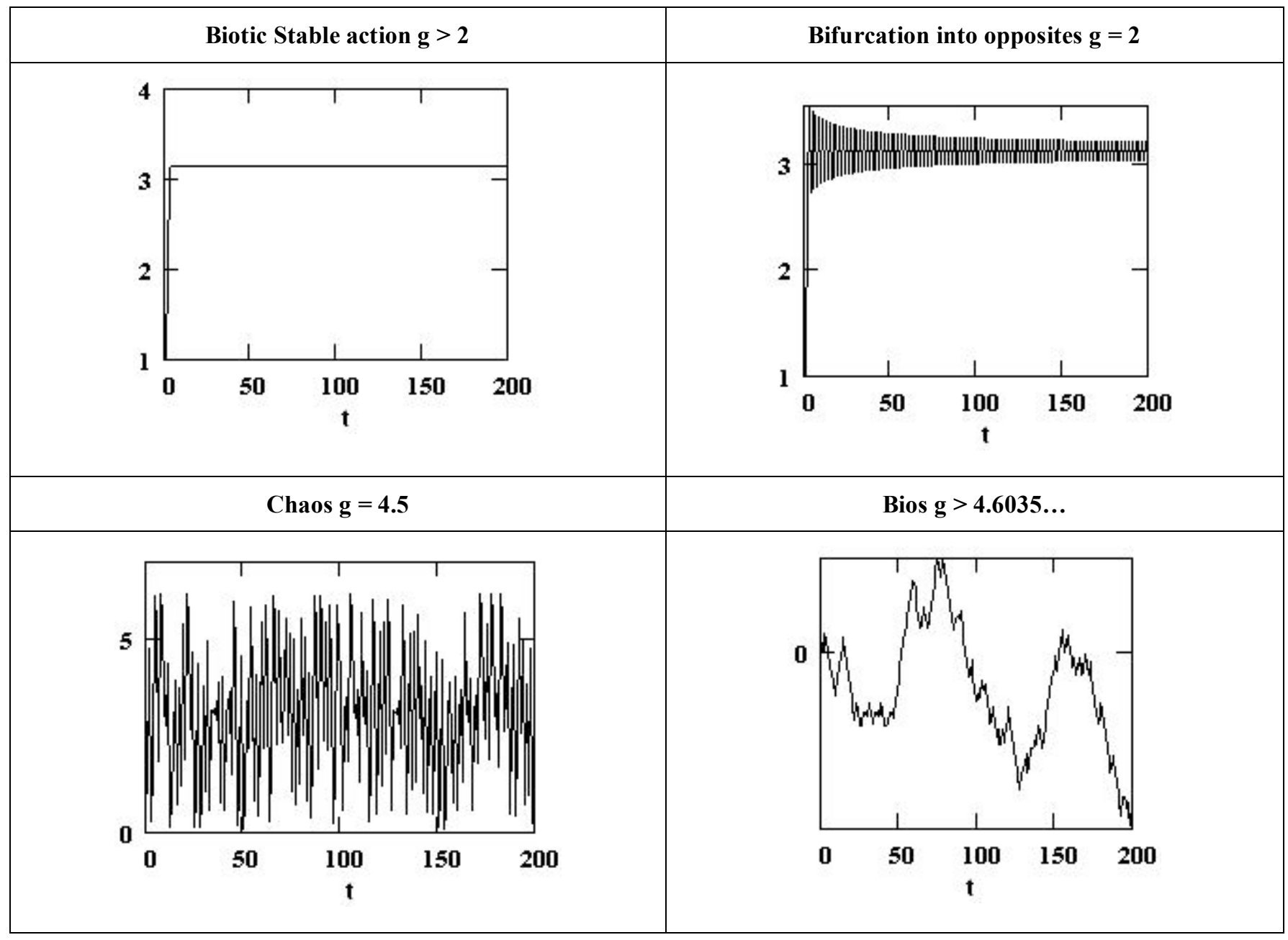

Fig. (4). Patterns generated by the process equation $[\mathbf{A}(\mathbf{t}+\mathbf{1})=\mathbf{A}(\mathbf{t})+\mathbf{g} * \sin (\mathbf{A}(\mathbf{t}))]$. Note the enormous difference in amplitude between Chaos and Bios. The transition from chaos to bios is abrupt, at 4.6035.

ration $[4,8,15]$. (7) Biology: population size of several animal species $[16,17]$. (8) Socioeconomic series (financial, employment, etc) [8, 9, 18]. (9) Music [19].

Table 1. Non-Periodic Processes

\begin{tabular}{|c|c|c|}
\hline & $\begin{array}{c}\text { Stationary Stable } \\
\text { SD Recurrence. } \\
\text { Stable complexity. } \\
\text { Flat power spec- } \\
\text { trum. }\end{array}$ & $\begin{array}{c}\text { Creative Diversi- } \\
\text { fication Novelty } \\
\text { Complexification } \\
1 / \text { f power spec- } \\
\text { trum. }\end{array}$ \\
\hline $\begin{array}{c}\text { Stochastic Random dif- } \\
\text { ferences between terms }\end{array}$ & RANDOM & $\begin{array}{c}\text { RANDOM } \\
\text { WALK }\end{array}$ \\
\hline $\begin{array}{c}\text { Causal Patterned series of } \\
\text { differences. Partial auto- } \\
\text { correlation }\end{array}$ & CHAOS & BIOS \\
\hline
\end{tabular}

Bios is not one more pattern to be recognized along with equilibrium, periodicity and chaos. Rather, the generation of novelty, diversity and complexity is an essential step in evolutionary processes. Its demonstration in Quantum Physics and in Number theory shows that Bios is a fundamental process. Bios is indeed everywhere. Neither chaos nor randomness, has ever been demonstrated in fundamental arith- metical or quantum processes; moreover many earlier claims of chaotic patterns in biology and economics have been later on disproven. Likewise many purported stochastic processes are now being recognized as "deterministic random walks", an oxymoron to describe Bios.

The demonstration in mathematical, physical and economic processes of biotic patterns previously found in clinical studies illustrates how medicine can contribute to basic sciences. Apparently Bios had not been recognized up to now as result of a focus on stability, invariance, and probability, and the consequent lack of methods to measure creative features.

\section{THE GENERATION OF BIOTIC COMPLEXITY BY SIMPLE PROCESSES}

Cardiac activity consists of unit actions (heartbeats) generated by the heart at a stable conserved pace that is continuously modulated by the brain through the sympathetic accelerating and parasympathetic decelerating nerves. This bipolar (accelerating and decelerating) feedback generates the biotic pattern of heartbeats. Bipolar feedback can be modeled with trigonometric functions. Such recursion (process equation) 


$$
A(t+1)=A(t)+g * \sin (A(t))
$$

generate biotic patterns [20]. Each value of $\mathrm{A}$ at the next time $t+1$, equals the value of $A$ at the previous time $t$ (conserved term) plus a change. The change term $\mathrm{g} * \sin (\mathrm{A}(\mathrm{t}))$ is $a$ feedback function of the ongoing process; $g$ is the feedback energy. Fig. (4) shows the patterns of steady action, bifurcation, chaos, and bios generated by this discrete equation - the corresponding continuous differential equation does not generate such complexity.

Consideration of the process equation (Fig. 5) indicates that the generation of biotic diversity, novelty, and complexity requires action, opposition, and conservation. The opposition must be positive and negative, synergic and antagonistic, sometimes increasing and sometimes decreasing the value of the action A. This is bipolar feedback [21], as contrasted to the classical positive or negative feedbacks. Only recursions of bipolar feedback generate Bios. Conservation is also necessary to generate bios; recursions without a conserved term such as $A(t+1)=g * \sin (A(t))$, generate only chaos, not bios, diversification and novelty. Unidirectional action, bipolar feedback and conservation occur at all levels of organization, probably accounting for the widespread occurrence of biotic and other creative processes. Energy continually flows and oscillates; matter and other structures provide conservation and stability.

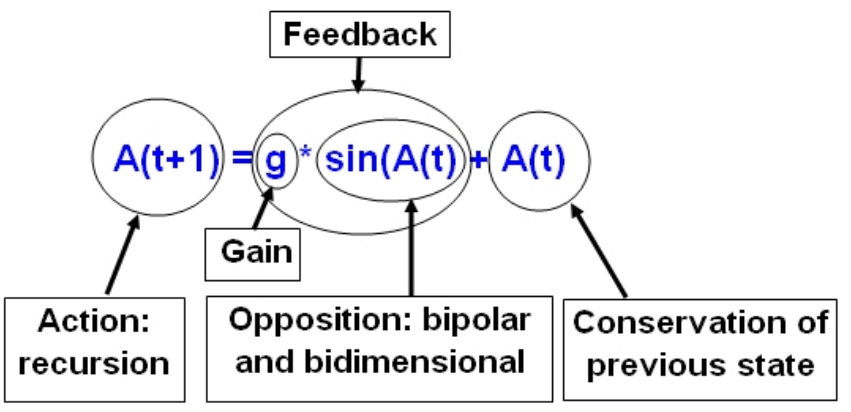

Fig. (5). Process equation.

\section{ACTION}

Communications are actions. Physical quanta, muscle contractions, economic transactions, and thoughts are actions. Life is a process; death is the cessation of change, while anatomical structure persists for a while. Repetitive actions (recursions), not static equations, generate patterns. All actions have essential properties in common: discrete units (as in digital computers), unidirectionality (asymmetry), frequency as energy, and quantitative, cumulative change.

At multiple levels of organization, actions are composed of units, as illustrated by sub-atomic quanta such as photons, heartbeats, economic transactions, sentences, and lives. And at all levels, actions are physical, i.e. changes of energy in time.

In quantum physics, energy $E$ is a function of frequency $f$ ( $\mathrm{E}=\mathrm{h} * \mathrm{f}$, where $\mathrm{h}$ is the Planck constant). As frequency is the inverse of time, there is an intriguing opposition between the two components of action.
Energy is a function of frequency also in the heart (its rate increases with effort and excitement) and in the brain (high energy mood and behavior correlate with faster changes in them), pointing to a fundamental homology across levels of organization.

A comprehensive survey of multiple levels of organization shows a positive relation between complexity and energy flow: the free energy rate density increases from galaxies to stars to planets to plants to animals to brains [22]. This relation, however, applies only to organized energy. The relation between temperature and complexity is nonlinear. Life occurs at the moderate temperatures of the planet, between the extremes of hot star cores and cold intergalactic space; normal brain function, the highest known complexity, occurs at $37^{\circ}$, between fever and hypothermia's coma.

In the process equation, complexity increases as the energy of the feedback gain $g$ increases (Fig. 6). Equilibriumlike, steady action occurs only at low energy. As energy increases, there are periodic oscillations, chaos, and bios which includes multiple leaps ('infinitation'). This sequence is consistent with the coexistence of stagnation with poverty, the occurrence of biotic patterns in healthy life-like economies, and their descent into chaos as a result of scarcity of goods and jobs. Counterintuitive as it may be, increasing, not reducing, energy will end chaotic disorder, and it does so by transforming it into creative biotic organization, not by generating equilibrium. We may thus expect that abundance can promote development, while austerity measures, as recommended by international financial institutions based on scarcity models of economics, push countries into chaos, as illustrated by economic disasters in Bolivia and Argentina in the early years of this century.

As defined in physics, action involves the accumulation of change. Notably, biotic patterns are generated by the (apparently) simple process of accumulating quantity. This is illustrated by the biotic pattern of the prime series -- as all other numbers, primes are generated simply by the addition of 1 . Numbers and number series abstract fundamental properties of action. Primes thus demonstrate the role changes in quantity in the generation of novelty and complexity [10], expanding the dialectic law relating quantity and quality.

Order is equally important. Time and causality are unidirectional. Causal novelty generates more change than shuffling: directed flow is more creative than undirected flux. Consideration of biological processes makes clear, as mechanics may not, why it is important to recognize that time is not reversible. The asymmetry of time is at the core of the creation of information.

\section{ASYMMETRY, NOT ENTROPY, MEASURES IN- FORMATION}

Action is change in energy, i.e. asymmetry within the flow of energy, and hence difference between two states. Difference embodies distinction and thus encodes information.

By analogy to the statistical mechanical model of entropy, Shannon defined a probabilistic measure of informa- 


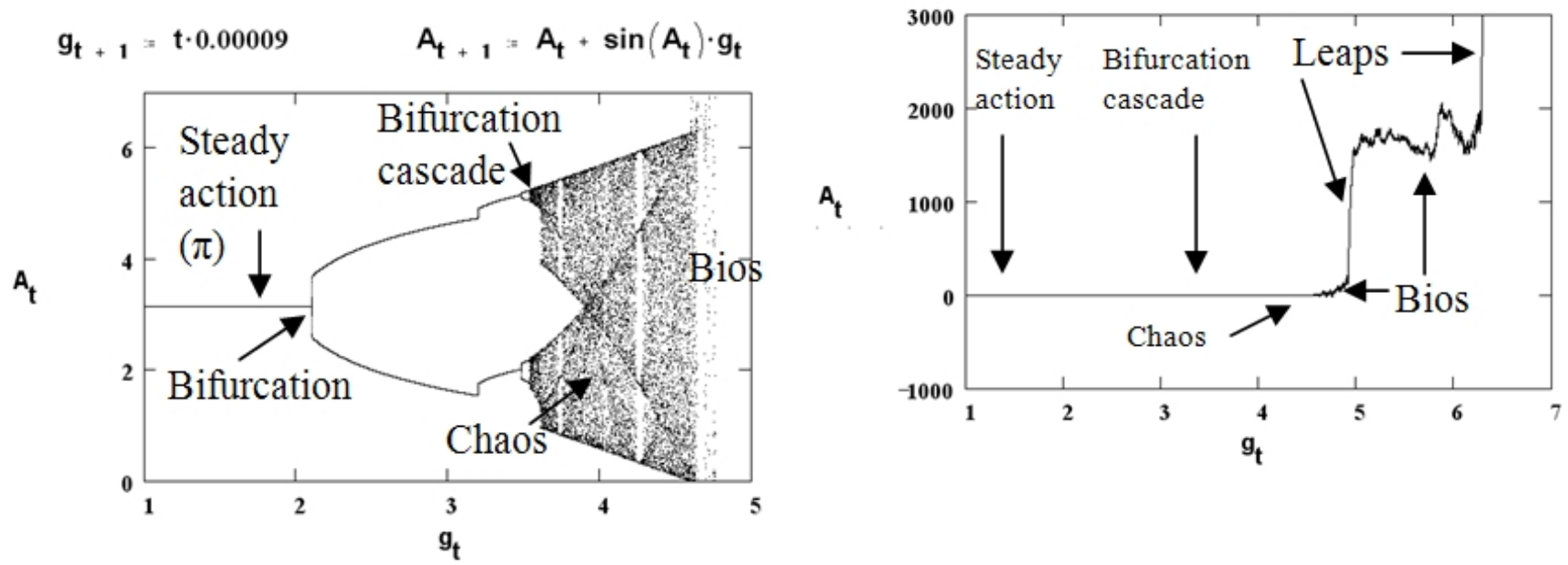

Fig. (6). Sequence of patterns generated by the process equation as the energy of the gain increases with time. Note the value $\pi$ of the initial steady action, the 'unifurcation' that follows the initial bifurcation at $g=\pi$, and the sharp transition from Chaos to Bios.

tion that he called entropy. This conception of information is useful regarding communication but it presupposes the existence of distinction and it does not address logical issues. In statistical mechanics, maximal entropy corresponds to perfect symmetry (equilibrium in all directions) often called randomness. Entropy is thus often said to be a measure of disorder. This cannot be true in the simplest case of onedimensional processes because the entropy of the series in its natural order, and that of its shuffled copy are identical. In any case, entropy is either information or disorder: it cannot be both. Also, disorder must be defined in terms of order. No such definition is offered by statistical mechanics.

Order is defined mathematically by Lattice Theory as asymmetry and transitivity. This is the definition of order that we can adopt to define information. Intuitively, information is contained in simple order (as in lattices) and in complex organization, (as in life). Indeed life requires a lowering of entropy, proposed Schrödinger [23] and Prigogine [24]. Notably, there is a decrease in entropy at the transition from chaos to bios in time series generated by recursions.

Let us examine what it is actually measured by Shannon's entropy by comparing biological data (necessarily complex) with mathematical models (evidently simpler). Focusing on change, let us consider time series, and measure entropy with a varying number of bins $[8,25]$. Such experiments show that statistical entropy actually measures the symmetry and the diversity of the distribution (Fig. 7).

The slope measures diversity and the intercept (at 2 bins) measures symmetry. Entropy is 1 for period 2 (symmetric and not diverse), maximal for random and chaos (symmetric and diverse). Natural processes and mathematical bios are asymmetric and diverse, so their entropy is less. Simple series are symmetric; complex series are asymmetric and diverse. For series with the same degree of diversity (slope of the increase in entropy as a function of the number of bins), entropy is lower for asymmetric complex processes than for simpler series. Thus information increases with asymmetry, while entropy increases with symmetry. Both information and entropy increase with the diversity of the series. Thus information is neither entropy nor negative entropy.

As a result of their asymmetry, biotic series have lower entropy than chaos. Likewise life has lower entropy than its environment because it occurs far from equilibrium [24]. Diversity and asymmetry, not entropy, portray life and measure information and complexity [8].

Notably, the mathematical definition of order as asymmetry fits biology well. Exemplary of complexity inference, Pasteur, based on the fact that biomolecules are always asymmetric (they deviate light rays to the left), reasoned that asymmetry must be present in the most basic physical processes [26]. The asymmetry in beta decay is exemplary [26], but more fundamental is the asymmetry of time and causality [1]. Physical evolution is clearly asymmetric, a fact described as 'symmetry breaking'. Without asymmetry, there can be no evolution and no complexity. Asymmetry is found at all levels of organization (see references in 8): the preponderance of matter over anti-matter, the optical rotation of atoms, the asymmetry of the crystals of which rocks are made, the ionic asymmetry across plasma membranes, anatomical asymmetries between left-right brain hemispheres, and power asymmetries of age, sex, class race, nationality.

Thus biology suggest a new definition of information: asymmetry is the simplest carrier of information. This is consistent with mathematics, as asymmetry is a fundamental property of order. Distinction is regarded as fundamental [27] but a boundary is not sufficient for distinction. There must be a difference [25] or asymmetry [8] between the opposite sides of the partition. Two values are necessary to carry information, but the two values must be asymmetric, such as $0<1$. In logic, false implies true but not vice versa. Indeed real opposites are asymmetric. Conceiving information and distinction as asymmetry rather than partition is a significant departure from the standard view of negation as classes of separate objects segregated by boundaries (set theory, traditional and Boolean logic). Directed graphs, where elements are connected by arrows, provide an intuitive and mathematical representation for asymmetry. 

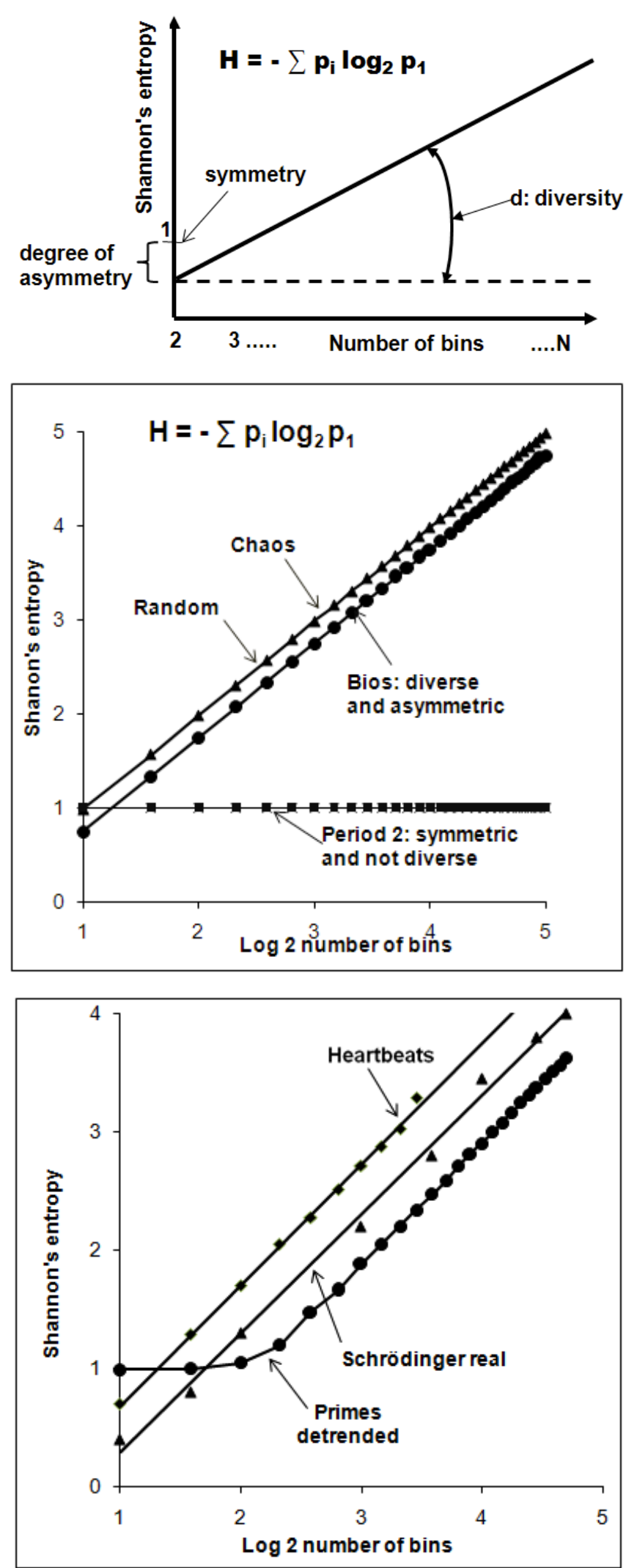

Fig. (7). Shannon's Entropy measured with an increasing series of bins. Top: schematic representation of the change in entropy as a function of the symmetry and diversity of the series. Middle and bottom: experimental results with empirical and mathematical time series.

\section{OPPOSITION AND MUTUAL FEEDBACK}

Change is the sine qua non of information. A constant interaction (e.g. the weight of our seating body) is not perceived. Moving a wire in a magnetic field generates a current. Information is embodied in the difference [25] or repetition [8] of two values. Opposition is universal: electrical charge, acid and base, biological sexes, complementary DNA bases, supply and demand, abundance and scarcity, cooperation and conflict, true and false. We walk with two legs, see with two eyes, and think with two hemispheres. Social roles often are paired: manager / employee, teacher / student, doctor / patient. Concepts are often paired (tall and short, content and form, quantity and quality). Opposites are partial, not diametric. Opposites overlap, as illustrated by quantum superposition. Opposites are similar, coexist, and complement each other, as remarked by many thinkers since Antiquity yet absent in binary logic. These properties are absent in the notion of mutually exclusive classes separated by boundaries. Notably, the pairing and interaction of opposites is 'recognized' by the 'brain computer': neural connections to opposite sides of the body are largely crossed. Also the logic of human-made computers needs to connect opposites. Opposition is the simplest symmetry; physics recognizes symmetries as fundamental. Noether's theorem states that each symmetry of a physical system implies that some physical property of that system is conserved (while others change), and conversely that each conserved quantity has a corresponding symmetry. But oppositions are also actions, and hence asymmetric. A focus on only symmetry and invariance is static. Paraphrasing Heraclitus, asymmetry is the father and symmetry is the mother who together create information and complexity.

Oppositions are coexisting and opposite asymmetric actions that together form a cycle of mutual feedback. Thus opposite processes form and transform each other: prey and predator adapt to each other; content determines form and vice versa; quantitative changes are accompanied by qualitative ones. Mutual feedback is an engine for change.

The unidirectionality of time establishes a direction in these cyclic engines. Mutual feedback does not replace causality by circularity (as sometimes claimed).

Opposites imply and antagonize each other. Their interactions are both harmonic and conflictual --bipolar feedback. For positive and negative polarities to coexist, they cannot be considered as polar ends in a linear continuum but must be plotted in two separate dimensions. Bipolar feedback is by necessity bidimensional. The harmonic (sinusoidal) functions that generate Bios mathematically are bidimensional. Likewise the activity of the opposing nerves that regulate heartbeats does not vary reciprocally, but may increase (or decrease) simultaneously. Their opposite actions must therefore be plotted in orthogonal axes (diamond of opposites (Fig. 8, top left). The coexistence, similarity and synergy of opposites can be represented rigorously by regarding opposites as orthogonal to each other.

Mathematical experiments show that bipolar sinusoidal functions generate bios. Harmony produces Bios. Organisms 


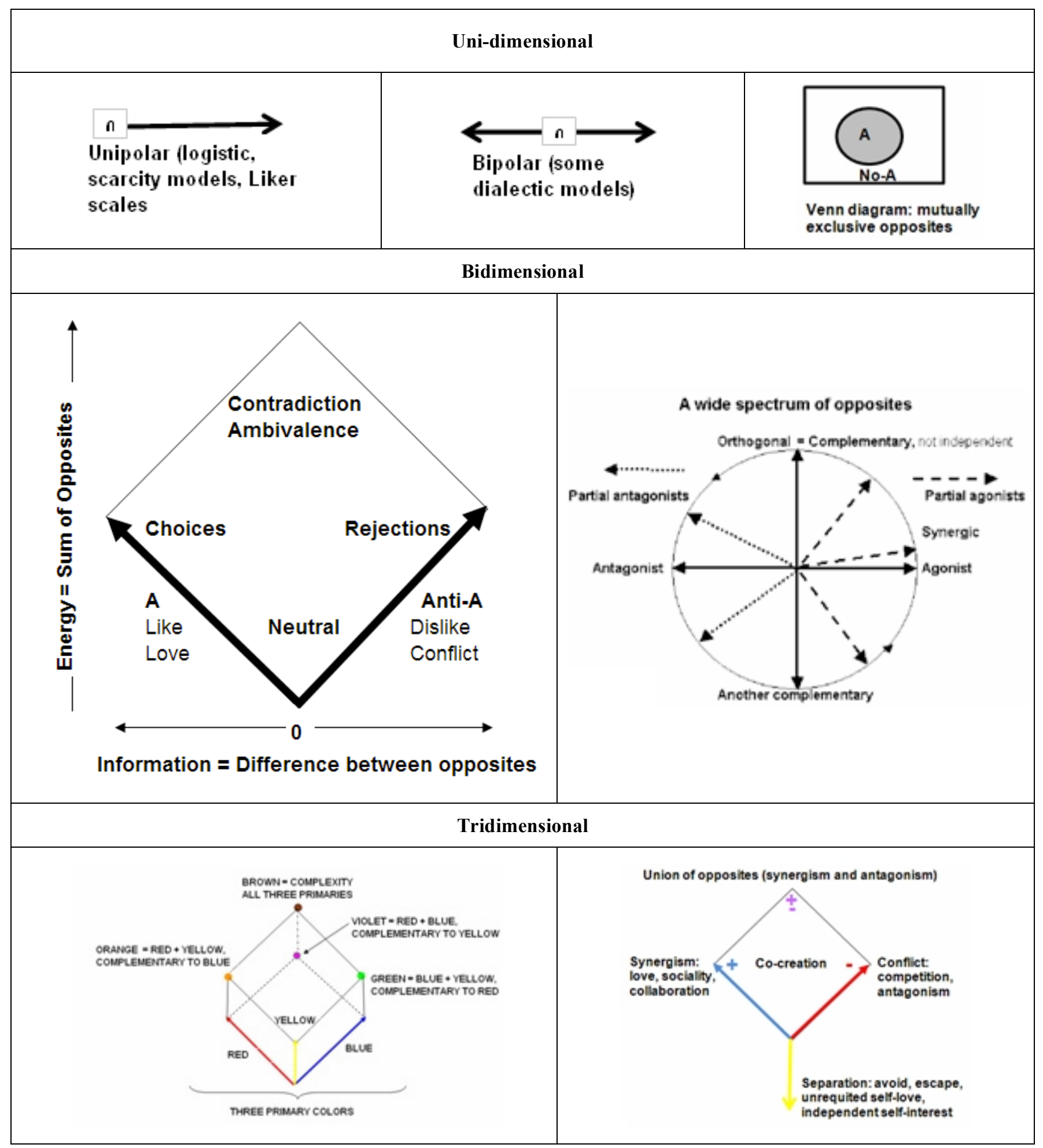

Fig. (8). Representations of oppositions.

survive because they fit with each other and with their physical environment. Mutual synergism selects what perpetuates. Co-creation more than competition, underlies the evolution of complexity. Symbiosis, endosymbiosis, multicellularity and sociality, are as important as the struggle of species in evolution $[8,17]$. History is moved by cooperation and solidarity not only by class struggle; progress results from production and abundance more than by competition and scar- city as portrayed by standard economics and by logistic models. Malthus and Darwin formulations assumed unipolar scarcity as the motor of change. But animal populations and socioeconomic processes both show biotic patterns of variation, indicating bipolar feedback. There is indeed synergism and antagonism among multiple species. Likewise economic processes are generated by the interaction of multiple pairs of opposites, such as supply and demand from multiple 
sources. There concomitant abundance and scarcity in almost every society, but in different respects and for different classes.

Contrary to standard binary logic, true and false are inseparable. Opposites coexist, and imply each other. They transform into each other, creating closed systems (as described by Group Theory). Just as every element of a group has one inverse, opposites in nature are multiple. There are cosmological, meteorological, biological, and economic cycles. Diametric opposites connect through intermediate steps in a cycle, not only through gradual transformation in a linear continuum. Paradigmatic are rotations, cycles of multiple opposites in which the transformations have directionality. Electromagnetic waves are helical. The helical pattern is also evident in proteins, DNA and RNA. Because material structures have volume, rather than being point-like, the application of mechanical forces usually generates rotation, not equilibrium, as astronomical bodies illustrate. The diamond of opposites allows one to see how both opposite actions provide energy (synergy, vertical axis) and yet oppose each other's information (horizontal axis) [8, 28]. Portraying bidimensional opposites in linear scales, as it is customary in sociological polls and psychological tests, irretrievably distorts the data [28]; conflictual opposites that create complexity are misrepresented as neutralizing each other.

We may advance further by considering how opposites relate to cyclic processes. Orthogonal opposites, such as $\mathbf{S}(\mathbf{t}+\Delta \mathbf{t})=$ $\mathbf{S}(\mathbf{t})+\Delta \mathbf{t} * \mathbf{C}(\mathbf{t})$ and $\mathbf{C}(\mathbf{t}+\Delta \mathbf{t})=\mathbf{C}(\mathbf{t})-\Delta \mathbf{t} * \mathbf{S}(\mathbf{t}+\Delta \mathbf{t})$ (where $=$ is approximate and $\Delta \mathrm{t}$ is a small number) generate an ellipse that is almost a circle, and the successive values of $\mathrm{S}$ and $\mathrm{C}$ are good approximations to the sine and the cosine [29]. A sine wave combines the unidirectional flow of action in time with the harmonic (sinusoidal) cycling of opposites, the arrow and the wheel. Circles, spheres and hyperspheres allow one to represent infinite pairs of diametric (linear) opposites and thereby orthogonal (complementary) and any other relation of partial opposites.

\section{COMPLEMENT PLOTS}

Waves can be decomposed into sine and cosine functions. Opposites can be detected in single time series by plotting the sine and the cosine of each term in orthogonal axes, and connecting successive terms with lines [30]. As sine and cosine are complementary opposites, the pattern observed reveals the opposites contained in the process.

The complement plots of few but significant processes, including heartbeat intervals [30], biotic series generated by the process recursion [30], prime number series [10], and series generated by the Schrödinger equation (unpublished) show a pattern of concentric circles (Fig. 9, left) spontaneously described as 'Mandala' by many observers. In contrast, random data generate disorganized uniform plots, and periodic and chaotic series generate simple patterns (Fig. 9, right). Mandala patterns indicate that the process is generated by a periodic process involving a limited number of nondiametric oppositions. Mandala symbols are found in almost all civilizations and appear in dreams and doodles. A Man- dala pattern is also observed in the two dimensional representation of E8 (Fig. 10), a mathematical object of 248 dimensions that appears to embody fundamental physical relations. The appearance of the same forms in natural processes and in psychosocial symbols points to a deep isomorphism between them. Complement plots demonstrate empirically the trigonometric dialectics of opposites.

\section{TRIGONOMETRIC OPPOSITES AND QUANTUM COMPUTATION}

Trigonometric models of opposition fit wave theories of nature, as well as the helical models of dialectic philosophy. Mathematically, bios is generated by recursions of sinusoidal functions that represent the cycling of diametric opposites that together form a circle. One may thus consider trigonometric functions to model the dialectic features of logical negation in a manner that can be implemented in quantum computers. This seems cogent. Helical electromagnetic waves physically embody information at the atomic, molecular, and biochemical levels, as well as in brain and computers. Electromagnetic energy embody two orthogonal oppositions. The coding of information requires two values (opposition) but actually orthogonal pairs of opposites embody information (four values) in natural processes. Qubits illustrate quaternity, i.e. orthogonal oppositions, in computation. A qubit can be encoded by the polarization of a single photon, any point in the sphere of opposites can thus be represented, and rotation gates be constructed [31].

Quantum processes involve continual change (rather than static states) which is causal (as explained by the Schrödinger equation), albeit often described as probabilistic. Position, spin, and many other properties are continually changing; this probably accounts for the observed superposition of opposite states (e.g. spins).

Instead of regarding the superposition of quantum opposites as a problem to be overcome in order to calculate according to the static concepts of standard logic, one may regard it as a way to generate dialectic logical functions. In this way we may adapt computer processes to the actual logic of nature, and thereby allow the full use of quantum processes for computation [32]. Current computers are constructed from electrical circuits connecting logical gates that represent Boolean logic functions. This corresponds to a mechanical, static, non-evolutionary world view in which opposites exclude each other, as they do in the case of abstract mathematical objects (principle of no contradiction). One may conceive of quantum computers that corresponds to a world view focused on processes, evolution and the creation of complexity via the interaction of opposites. As quantum processes generate biotic patterns, one may develop quantum devices as logical gates that embody mutually transforming opposites.

Three essential tenets of Aristotelian Logic have been consistently ignored by later thinkers: (1) the existence of a third open value (neither true nor false) for what has not yet come to pass, thus allowing for creativity; (2) the superiority of states of coexisting opposites (the "Golden Middle"); and 

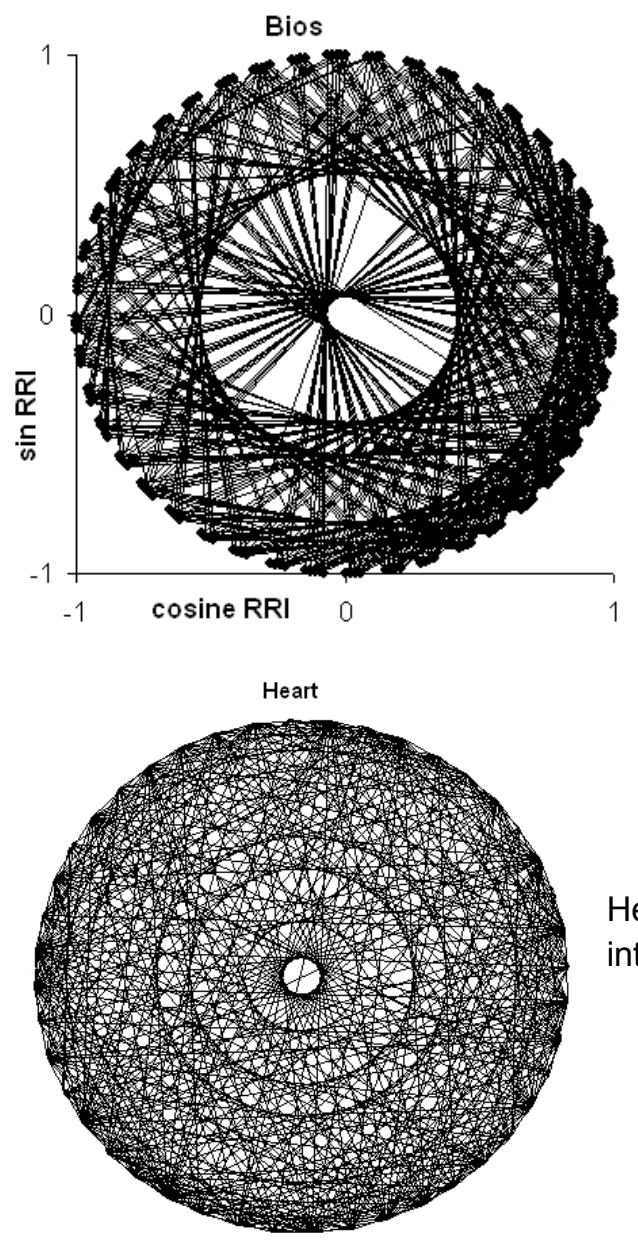

Heartbeat

intervals

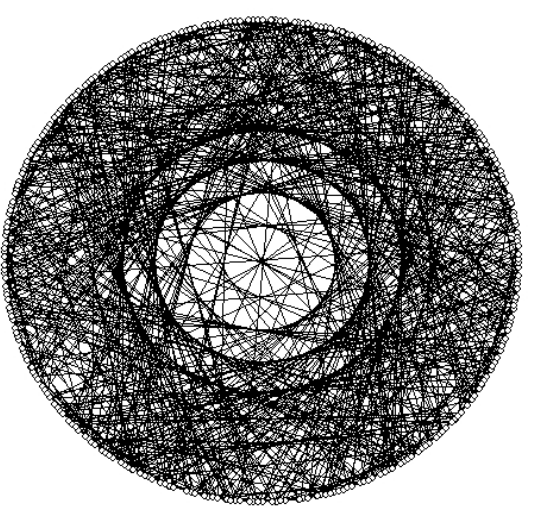

Prime numbers

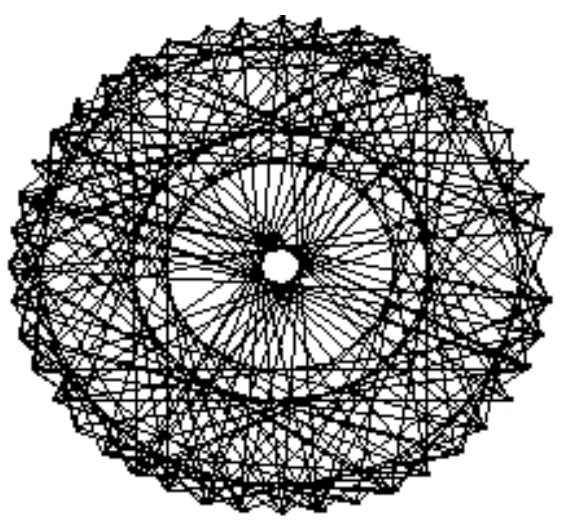

wave

function
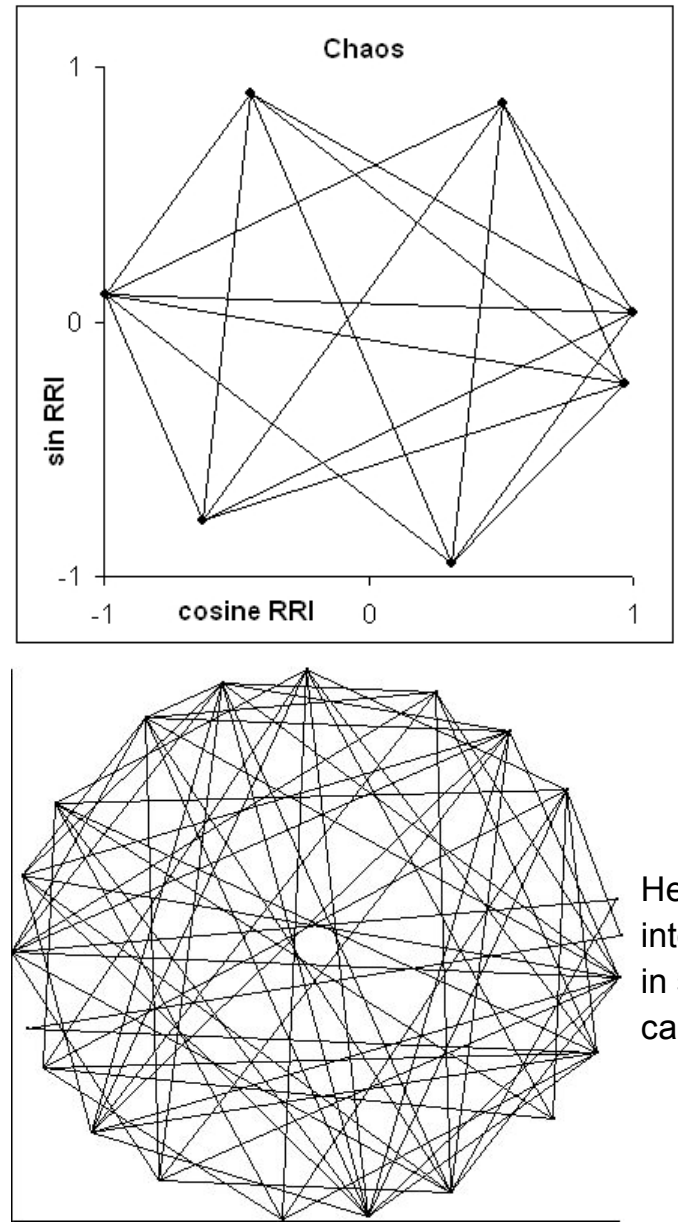

Heartbeat intervals in severe cardiac failure

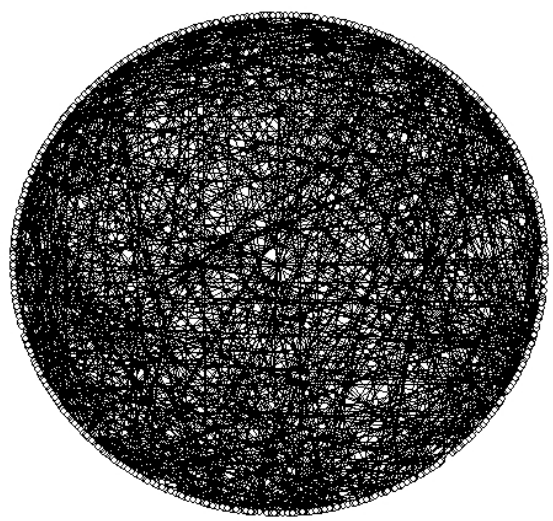

Random

numbers

in ascending order

(as primes)

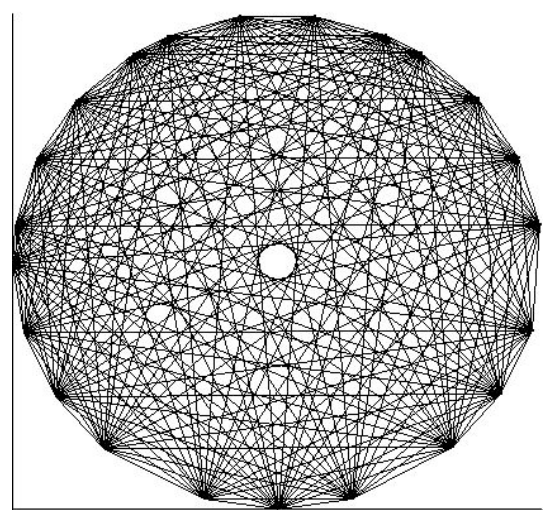

Random

integers.

Equidistant

Fig. (9). Complement plots. Mathematical biotic and chaotic series have been rounded to nearest integer. The prime numbers Mandala demonstrate the basic nature of the rotation of opposites. The disappearance of the Mandala pattern in severe cases of cardiac illness indicate that it is biologically significant. 
(3) the local character of the principle of no contradiction: Opposites do not coexist at the same time, at the same place and in the same context. For instance, opposite sexes coexist in time and are separate in space, and the same person is first young and then old. Computational logic may be expanded by including these essential tenets of Aristotelian logic. Boolean logic and its realization by electrical circuits gave us the computer revolution but Informatics may progress further by attending to both the physics of nature and the psychobiology of thinking.

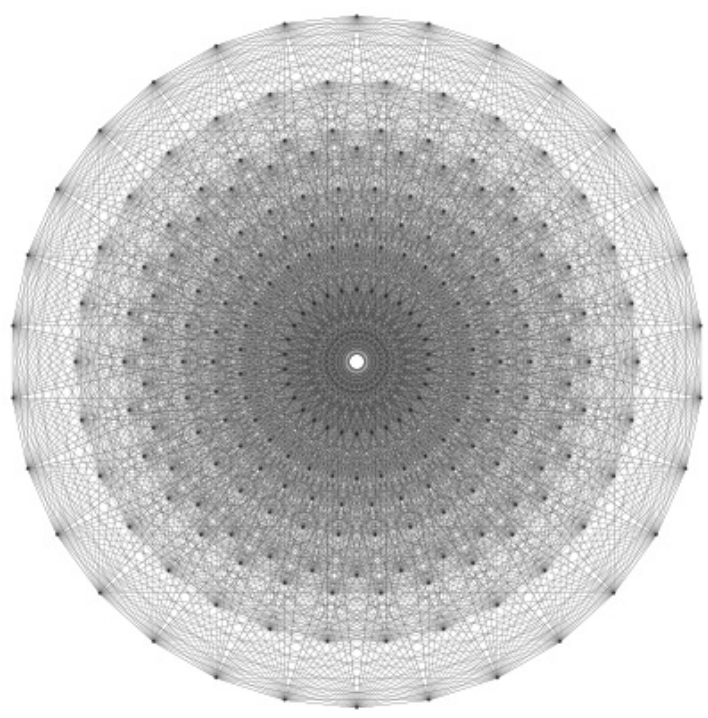

Fig. (10). A Mandala-like pattern of concentric circles in the two dimensional representation of E8.

\section{TRIADIC STRUCTURE AND INFORMATION}

Matter transmits, conserves, and transforms information. Brains and computers are material structures. Matter itself is structure, the asymmetric distribution of condensed nuclei of mass. Just as opposition consists of two oppositely directed asymmetries, structure is asymmetry in three dimensions. Macroscopic matter has three dimensions. This tridimensional structure represents a minimum of complexity. Many behavioral, emotional, social and conceptual categories are triadic (e.g. fight, flight or surrender; thesis, antithesis and synthesis). Biological organisms cooperate, compete and avoid each other. Avoidance is likewise significant in social processes -hence emigration as an alternative to scarcity and struggle. Information itself is triadic: there is not only ignorance and uncertainty, but also misinformation.

Hierarchies of complexity and of power display another fundamental triadicity, as each level has one below that has temporal priority and one above that has supremacy of control (see later).

There is an as yet more fundamental triadicity: atomic nuclei are formed by the nucleation of quarks of three polarities and their opposites. A triadic organization of three primary factors (and three secondary opposites) capable of combining in multiple ways is also observed in visual colors. Each primary color has a complementary opposite equal to the sum of the other two primaries, and this set of colors can combine in various ways to create a limitless number of new colors, including complex browns that include all three primaries. It is this combinatorial power, not just the existence of three values, that is creative.

There are dire consequences to the binary logic that dominates the framing of scientific and social problems. Black and white thinking fosters fanaticism and social conflict, as well as neurosis and depression (see multiple psychiatric references in [8]), while thinking in color, or at least in shades of gray, has been shown clinically to be is therapeutic. A chromatic, or at least a triadic model for information seems desirable.

Quantum chromodynamics suggests a tridimensional chromatic logic $[1,8,32]$, beyond uni-dimensional models (scarcity models of biological and economic competition) and two-dimensional dialectics. The concept of information merges naturally with that of color structure, as difference encodes information and the recursion of differences creates three pairs of opposites, as illustrated by arithmetic (Fig. 11, top) and trigonometric recursions (Fig. 11, middle and bottom).

The trefoil knot, the simplest nontrivial knot, an icon of topology, the third mother structure of mathematics, embodies six crossings, alternatively over and under (Fig. 12), which one is tempted to connect to the basic three pairs of opposing colors in a chromatic structure.

\section{DIMENSIOGENESIS: GENERIC FORMS AND CREATIVE DEVELOPMENT}

Creativity (not complexity or uncertainty, pet subjects in this decade) is the core process to be studied to understand cosmological and biological evolution from simple origins. Random origin is not simple; in fact randomness has maximum algorithmic complexity [33].

The unipolar, bipolar and tripolar dimensions of action, opposition and space appear to be the minimum of complexity present in even the simplest natural processes. Time, and hence action, is unidirectional, i.e. it is unipolar, it displays one asymmetry. Electromagnetic energy involves orthogonal pairs of bipolar opposites that generate circular symmetry $\left(2^{\infty}\right.$ diametric opposites) which actually is a rotation (circular asymmetry). The nuclear forces that create and conserve tridimensional matter are tripolar. We thus find oneness, twoness and threeness, three generic forms according to Pythagoras, Pierce, Gödel and Jung.

Notably, there is also a parallel with Lattice, Group and Topology, the three mother structures of mathematics [34] which also coincide with basic cognitive structures [35], highlighting the homology between levels of organization. These mathematical disciplines describe uni-directional asymmetry, twoness or inverses (opposition), and spatial form, which is tridimensional in nature and in knots -a core subject of topology.

These generic forms must per force participate in natural processes and evolution. Indeed unipolar gravity, bipolar electromagnetic energy and tripolar nuclear forces exemplify the materialization of such abstract forms. These forms are 

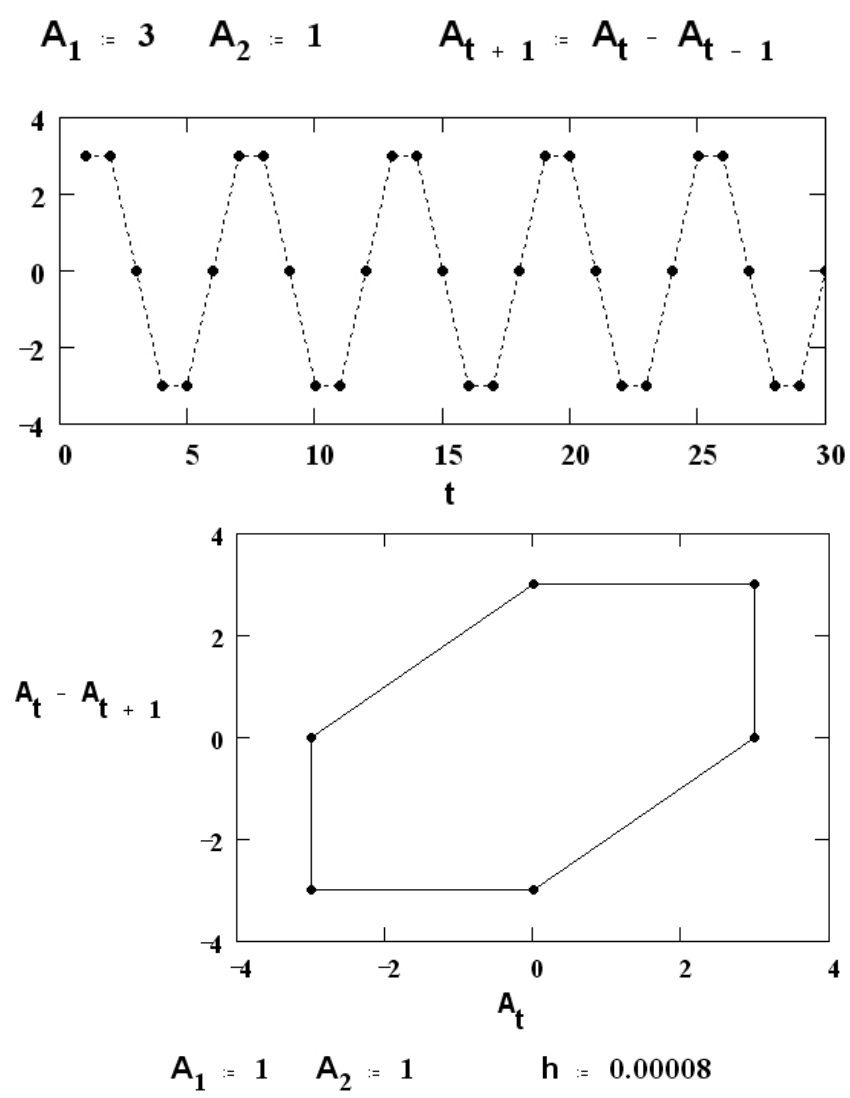

$A_{t+1}:=A_{t}-A_{t-1}+\cos \left(A_{t}\right) \cdot h \cdot t$
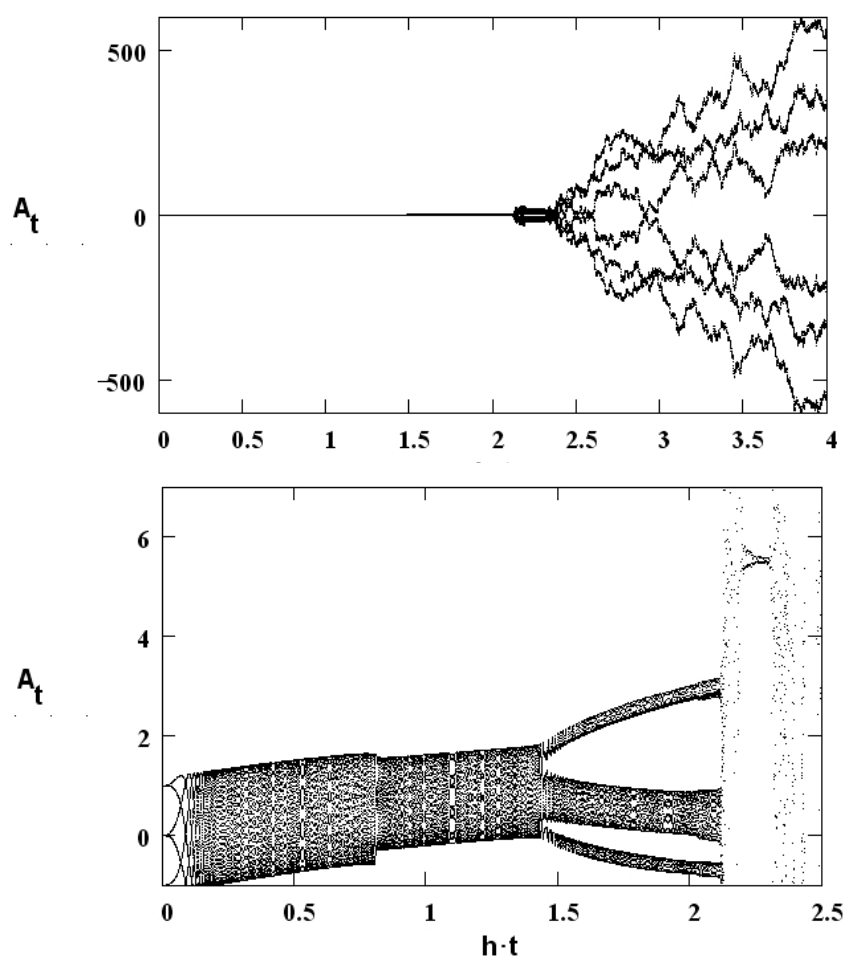

Fig. (11). Hexadic patterns in recursions of differences between consecutive terms. Top: recursion of the difference $A(t)-A(t-1)$ generate period 6 as shown in phase plane; $\mathrm{A}(\mathrm{t}-1)-\mathrm{A}(\mathrm{t})$ does not. Middle and bottom: recursion of difference and of trigonometric function complex periodic patterns and chromobios (a biotic pattern with six starnds).

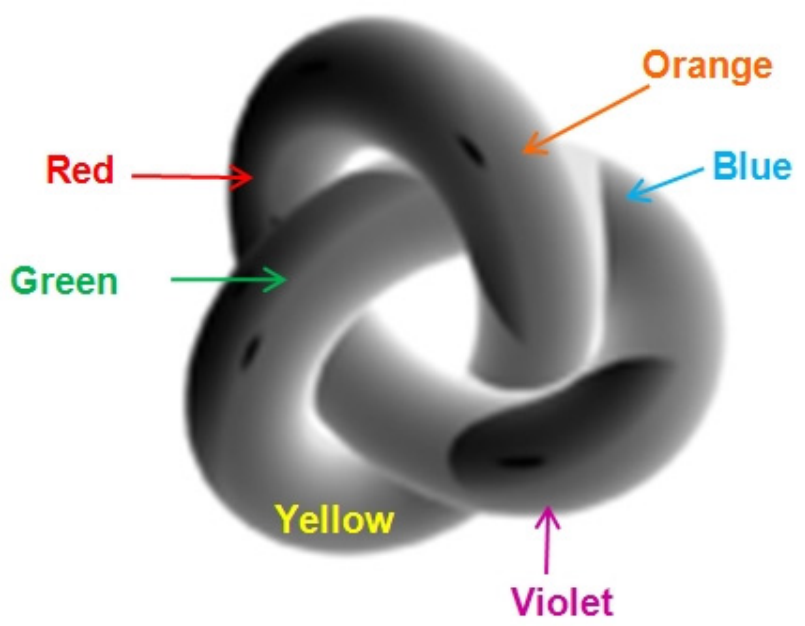

Fig. (12). Trefoil knot. Arbitrarily assigning a color one crossing over and labeling successive crossings according to frequency shows the alternation of primary and secondary colors and the pairing of complementary colors. This way of writing the pattern of a knot by naming the undercrossing and overcrossing sites in order of traverse) is the "Dowker Code", much-used in making tabulations of knots and links.

also embodied at the molecular level (e.g. Pasteur's asymmetry). Likewise the human body manifests these forms in its back-front asymmetry corresponding to locomotion (action), its right-left symmetry, and its vertical hierarchy (see later) $[1,8]$.

Exemplary of complexity inference, Aristotle provided a biological model of change, now employed to describe social, economic, psychological and scientific development. Embryonic development is paradigmatic of causal and creative evolution. External interactions can affect and even stop it, but not fundamentally change its outcome -frog eggs always generate frogs. In the same manner, cosmological, chemical and biological evolution may be the creative outcome of simple generic forms. As mathematical relations are logically necessary, physical processes cannot do other than embody them. Thus Bios theory regards unipolar action, bipolar opposition and tripolar structure as necessary and sufficient generic forms to engender complexity, leading to the development of multiple levels that interact with each other in mutual but hierarchical feedback -one side has priority and the other supremacy (see later).

Multiple pairs of opposites are generated by bifurcations in recursions, 'symmetry-breakings' in physics, and differentiation in biological development. Bifurcations (forking into opposites) are notable in biological processes and structures as illustrated by cell divisions, arteries, neurons, bronchi; trifurcations are rare, but observed in plants. Bifurcation generates opposites. Bifurcation trees generate multiple $2^{\mathrm{N}}$ opposites and thereby complexity (chaos, bios, etc). In recursions, bifurcation cascades generate period 3 , and period three implies infinite harmonies (Sarkovskii's theorem [36]).

Conversely, multiple oppositions generate bifurcations, as in the feedback processes illustrated by the logistic and process equations. In mathematics, the dialectic of multiple proofs and refutations develops insight [37]. In nature, mul- 
tiple oppositions are cyclic engines of change, generating catastrophes, bifurcations, and complexity. Electromagnetic waves bond structures -atoms, molecules, organisms. Anabolism and catabolism in living organisms, and photosynthesis and respiration in the biosphere, illustrate the creative cycling of opposites. The cycling of water constructs the surface of the planet.

Complexity may thus be generated by 'mathematical engines', so to speak, such as the accumulation of actions modeled by addition that generates primal novelty and infinite harmonic multiples; spiral and helical generators such as trigonometric functions and the Fibonacci series; unipolar logistic scarcity that generates one level of chaos; bipolar logistic recursions that generate multiple levels of chaos [8]; bipolar feedback that generates bios; and of course more complex engines.

\section{SIMPLICITY AND CAUSALITY}

A creative process may be expected to display low dimensional components representing the processes that generate it, and high dimensional components that display its complexity. The quantification of complexity is not sufficient. It is necessary to identify the factors that generate complexity. Contrary to oft spoken statements, random changes cannot produce anything. Random series do not show diversification, novelty or temporal complexity. Causal, temporally directed action, not randomness, is the fundamental simplicity that generates complexity.

What looks disorganized in one dimension can reveal pattern in two-dimensions as illustrated by phase planes, return maps, diamonds of opposites and complement plots.

Dimensional simplicity and complexity can be measured by examining various features of a process at $1,2,3, \ldots \mathrm{N}$ embedding dimensions, thereby revealing both its simple and complex components, as illustrated in embedding plots (Figs. 1 and 2). Current 'embedology' purports to determine the dimension at which a process must be studied. As illustrated in Figs. (1 and 2), studying multiple embeddings do not distort the data and allows one to study both the complex process under consideration and its simpler roots in one, two, three and higher dimensional factors. Unipolar action, bipolar and bidimensional opposition, and tripolar and tridimensional structure occur at all levels of organization.

Generic forms reoccur in multiple orthogonal dimensions and create new and complex forms. This homology generates fractality, but fractality, is defined in one dimension (scale) and focuses on repetitive form.

Asymmetry, opposition and tridimensionality coexist at all levels. There is one, two and tridimensional asymmetry. Every action occurs in tridimensional space. There are three pairs of opposites in the three dimensions of space. Opposites coexist in one unipolar dimension: Einstein's principle of equivalence may be interpreted as the identity of gravitational mass that accelerates falling bodies and the resistance to acceleration provided by inertial mass. Gravitational and inertial mass are detected in different experiments, but they manifest together in the fact that all objects fall at the same velocity (Galileo). Unipolar processes are their own oppo- site; the opposites are identical. This surprising conclusion derives from empirical science; it flows from physics to logic, not from philosophy to physics. Providing a rationale for what in Newtonian mechanics is a coincidence also supports the dialectic tenet that opposition is universal.

\section{INFORMATION AS ASYMMETRY IN 1, 2, 3..., N DIMENSIONS}

Information is created by action, not by symmetric flux (e.g. 'randomness'). Actions transmit information. Actions have consequences There cannot be isolated independent events, isolated from the preceding actions and having no consequences, as often postulated in statistical modeling. Actions are generated by causal mechanisms, such as the 'mathematical engines' discussed above.

Regarding information as a function of our knowledge leads one to the calculation of probabilities. Regarding information (and complexity) as an abstraction of objective properties of processes and structures leads one to measure them in terms of dimensions. The mathematical apparatus to describe objects of any number of dimension is available. The universality of physical dimensions does not mean that there are no more complex ones. Only a reductionist perspective limits dimensions to physical dimensions. By focusing on the universal, physics does not deal with dimensions of form, which are central in biology. In-form-ation is form. Fractal dimensions illustrate the use of dimension to define form.

The evolution of complexity may be conceived as the creation of forms of higher dimensions --creation as dimensiogenesis. Formulating logic in terms of dimensions can lead us beyond the static logic of classes.

Information is order in many dimensions. The simplest order is given by temporal sequence. Equally universal is order in two dimensions as processes invariable involve pairs of orthogonal opposites. The three spatial dimensions of material structure are likewise universal. Processes and structures are also organized in more complex topologies the description of which requires multiple dimensions of form beyond the standard three physical dimensions. These considerations suggest that information is asymmetry in $1,2,3$, $\ldots \mathrm{N}$ dimensions. Information is embodied in the three mother structures of mathematics -Lattice, Group and Topology. Information is determined. Information is mathematical form.

Nature is mathematical at all levels of organization (Galileo). Information is always embodied in energy or matter (Shannon [38]). Form is embodied in matter (Aristotle), thus information is creative. For Aristotle, everything that exists must have an origin, so he postulated an uncreated creator. Matter is neither created nor destroyed, but only transformed, proposed Engels. Energy becomes matter in the evolution of the universe, demonstrated $20^{\text {th }}$ century physics, starting with Einstein. Space is not empty but in continual flux, as virtual particles emerge and annihilate each other. Mathematical form relations are logically necessary, and thus give form to flux $[8,39]$. Information morphs flux into flow. Information creates free energy out of uncertainty 
(flux). For instance, iron atoms can spontaneously line up their magnetic directionality upon cooling.

\section{PRIORITY, SUPREMACY AND COMPLEXITY INFERENCE}

As the oldest profession and the oldest science, medicine attends to the most fundamental problem, life. Also, a medical perspective is especially valuable by underscoring the need for comprehensiveness. A medical perspective does not replace physics, mathematics, sociology or psychology but integrates them. Likewise Informatics must integrate multiple processes.

How to integrate multiple disciplines? Mutual and hierarchical feedback in neural regulation provides a concrete model (Fig. 13). In the regulation of cardiac actions, the neural feedback is not only bipolar but also hierarchical: the heart supplies energy (oxygen and foodstuff) to the brain, a function that has priority; while the brain provides information to the heart about the needs of the organism, a function that has supremacy.

This dual relation of priority : supremacy [1, 40] also obtains for the Central Nervous System levels (cortical, diencephalic, mesencephalic, spinal), where each level receives the input and modulates the function of those below (as described by the $19^{\text {th }}$ century British neurologist H. Jackson), with the brain cortex having 'supremacy' over all others (as described by the Russian neurophysiologist I. Pavlov). The functions of these Jacksonian levels of the brain correspond to levels of complexity among levels of organization in nature (Fig. 13, bottom). As every level of organization exists between simpler and more complex ones, upward and downward causation occur in the context of a bidirectional and hierarchical feedback.

The concept of hierarchical but bidirectional interactions between levels of complexity provides a scheme to integrate various scientific disciplines: physics and chemistry serve as foundations, and deserve priority as explanations of complex processes; at the other end, sociology and psychology provide goals, guidelines and critical evaluation of assumptions and methodology. Research walks on two feet, reduction and complexity inference.

The studies discussed here illustrate the usefulness and limitations of complexity inference. The homology between levels of organization, and the supremacy of complex processes over simpler ones validates it as a heuristic strategy but do not provide certainty. In the midst of uncertainty, we need further guidance.

Here enters the unique aspect of medicine, its therapeutic, i.e. humanistic ethics. In Informatics, 'first do no harm' means not to develop robots to fight war against people or promote 'models' of economic processes that promote poverty. Scientists also have special responsibilities as educators of the public at large in modern societies, where people regard us as custodians of the truth. These are not just ethical questions; they also have to with the substance of science itself. This is illustrated by medical practice when it becomes dominated by a pharmaceutical industry that has repeatedly marketed drugs that actually foster cardiovascular illness, diabetes, and strokes. Any science may lose its bearings when it becomes disconnected from the implications of its 'models'. Abstracting communication from the physical processes that give rise to it, the application of Informatics to psychiatry [25] actually led to depriving psychotic patients of needed biological treatment and blaming parents for their unfortunate circumstances. Logic has practical applications and human implications. Our 'models' must abstract deepseated properties of reality.

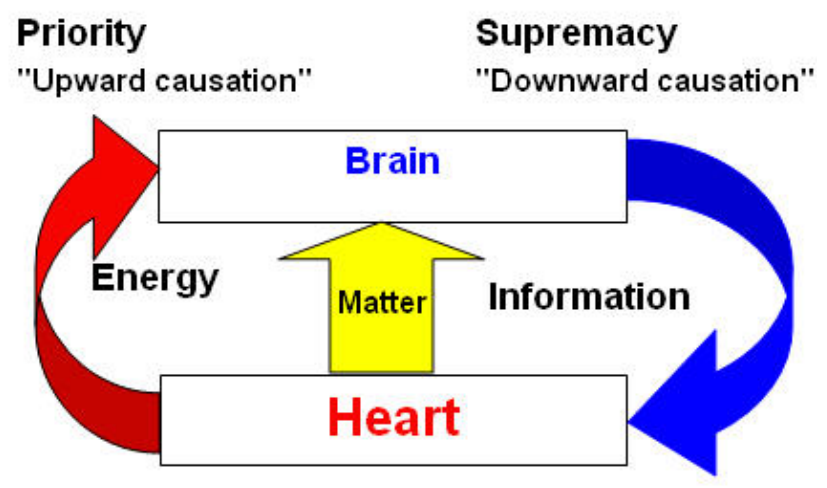

Fig. (13). Hierarchical feedback. Top: the priority / supremacy loop is illustrated by the heart which supplies oxygen and foodstuff to the brain, while the brain provides information to the heart determining its rate. Bottom: the more complex level has greater density of information [22] and the simpler level has greater extension (energy and matter). This establishes a hierarchy of levels of organization (bottom right).

Attending to the human significance of our scientific activity guides to new findings even in apparently specialized fields. Consider again heartbeat series. Their study led to interesting findings because it is a clinically significant, vital phenomenon -the study of insignificant processes is more likely to lead to insignificant findings. Considering how heartbeat intervals change in active humans revealed features of creativity not obvious when studying invariant laboratory conditions. It leads us to a highly relevant measurement of complexity, temporal complexity. While other definitions of complexity are applicable in different contexts, temporal changes in pattern are a most essential feature of creative processes. Considering variation, instead of assuming random variability, revealed Bios. Awareness that the organism functions as a whole system [41] under the control of the central nervous system led to consider systemic causation rather than a multitude of hypothetically independent factors. Recognizing the mutuality of causation led to focus on feedback [42] and the opposing cardiac actions of sympathetic and parasympathetic nerves. Dialectics [1, 43] led us to searching for opposites, leading to the finding of Mandala archetypes in heartbeat, prime, and quantum function series. Considering facts does not weaken the objectivity of the research. On the contrary, objectivity is interfered with by choosing invariant laboratory conditions, assuming variability, focusing on heart rate (an average that erases variation), presupposing that there are many independent factors that affect heart rhythms, and attempting to reduce complexity to simplicity and to account for creative phenomena with stochastic or deterministic models. Finding creativity in clini- 
cally significant processes led to the hypothesis that creative life-like processes are also fundamental in mathematical, physical and economic processes.

Science often contributes to misinformation through often unconscious ideology -not obvious religious or political ideology, but static, abstract, reductionist, and mercantile perspectives that make scientists attempt to reduce creative processes to deterministic mechanics and to see all interactions, from symbiosis to grooming [44] (i.e. macaque caresses) (!), as market transactions, while at the same time believing, in good faith, on their own scientific objectivity.

Scientists regard a study as completed when it provides an answer that fits their conception of reality --for instance, Darwinist zoologists repeatedly publish evidence for competition, and ignore symbiosis, mutualism, and group selection. 'Mathematical' economic models that lead to poverty, deforestation, and pollution are not economic, or scientific. Biological evolution and economic development hinge on creative processes. Thus humanistic intent is not a moralistic goal that could distort scientific objectivity, but a guide to lead us to seek what is fundamental. The purported independence of 'objective' science does not reflect ideological neutrality but lack of psychological insight. The cognitive, emotional and pragmatic aspects of mentation are inseparable and interactive [45].

\section{THE LOGIC OF NATURE AS A MODEL FOR COMPUTER LOGIC: A PROJECT}

Often information is defined as entropy, entropy is regarded as disorder, quantum flux is described as uncertainty, innovation is attributed to independent chance events, and selection is ascribed to competition. Pursuing clinical studies to their ultimate consequences, there is evidence to regard information as order, not entropy, entropy as symmetry and diversity, not disorder, complexity as temporal, quantum uncertainty as flux, and causal action rather than chance events as the origin of innovation.

Logic derives from Logos -Heraclitus' formulation of the reason contained in natural processes [46]. In this tradition, Aristotle founded Logic, Engels formulated a 'Dialectic of Nature' [47], Boole formulated mathematical logic as the 'Laws of Thought' [48] (not as pure mathematics), and Shannon [38] showed how these laws are embodied in electrical circuits, thus breaking the ground for Informatics. In this tradition, it behooves us to develop it further to incorporate the dialectic and chromatic features evident in quantum physics, social processes, and human reasoning. Such a three tiered logic would integrate Boolean, Biotic and Chromatic models.

Uni-dimensional Boolean logic is linear, and unipolar. It abstracts the fundamental asymmetry $0<1$, i.e. lattices. It is at the core of set theory and it is applicable not only in extension but also in other hierarchies

Bidimensional Biotic Logic: The helical form of electromagnetic energy -the carrier of information - as well as many other natural processes point to dialectic logic. Dialectic logic must be mathematized $[49,50]$ as verbal dialectics is useless for computation and prone to political distortions $[1,8]$.
Group theory abstracts some properties of dialectic logic [8, 49]. Bidimensional and bipolar trigonometric functions abstract the helical structure of dialectics. Mathematical recursions show that self-referent dialectic helices generate biotic complexity, just as electromagnetic energy creates complexity in nature. Beyond dialectics, a Biotic Logic is to be developed. Tridimensional Chromatic Logic: Beyond processes, we need to consider structure, and thus consider synthesis as in chemistry. The formation of a system is the combination of diverse parts that creates complexity, not a loss of diversity in the union of opposites. (In a dialectic period 2, the third stage is not a synthesis in the sense of a chemical synthesis, but another element of the same color as the thesis.) Topological dynamics provides scientific bases for the development of a logic that is structural and chromatic. To reason is to follow the logic of nature -nothing else can be reasonable. Informatics must adopt the logic of nature. Human and computer logic must be empirically based.

\section{ACKNOWLEDGEMENT}

I am deeply grateful to Mrs. Maria McCormick for her generous research support, as well as to doctors Louis Kauffman, Gerald Thomas, Warren Grimsley, Linnea Carlson-Sabelli, and Keelan Kane of the Society for the Advancement of Clinical Philosophy for enlightening discussions of this article.

\section{REFERENCES}

[1] H. Sabelli, Union of opposites: A comprehensive theory of natura and human processes. Brunswick Publishing, Lawrenceville, VA, 1989.

[2] M. Malik and A. J. Camm, Heart rate Variability. Futura Publishing, Armonk, NY, 1995.

[3] K. G. Dalton, G. S. Dawes, and J. E. Patrick, "Diurnal, respiratory, and other rhythms of fetal heart rate in lambs", Am. J. Obstet. Gy necol., vol. 127, no. 4, pp. 414-424, February 1977.

[4] L. Carlson-Sabelli, H. Sabelli, J. Zbilut, et al. "How the heart informs about the brain: A process analysis of the electrocardiogram". Cybernetics and Systems 94. Ed. R. Trappl. World Scientific Publishing Company, Toh Tuck Link, Singapore, 1994.

[5] H. Sabelli and A. Abouzeid, "Definition and Empirical Characterization of Creative Processes", Nonlinear Dynamics Psychol. Life Sci., vol. 7, no. 1, pp. 35-47, January 2003.

[6] H. Sabelli, "Novelty, a measure of creative organization in natural and mathematical time series", Nonlinear Dynamics Psychol. Life Sci., vol. 5, no. 2, pp. 89-113, April 2001.

[7] W. Berry, Home economics. North Point Press, San Francisco, CA, 1987.

[8] H. Sabelli, Bios: A Study of Creation. World Scientific, Toh Tuck Link, Singapore, 2005.

[9] M. Patel and H. Sabelli, "Autocorrelation and frequency analysis differentiate cardiac and economic bios from 1/f noise", Kybernetes, vol. 32, no. 5-6, pp. 692-702, November 2003.

[10] H. Sabelli, The Biotic Pattern of Prime Numbers Supports the Bios Theory of Creative Evolution From Radiation to Complexity. International Conference on Complex Systems, 2007.

[11] L. H. Kauffman and H. Sabelli, "Riemann's Zeta Function and the Prime Series Display a Biotic Pattern of Diversification, Novelty, and Complexity", International Conference on Complex Systems, 2007.

[12] H. Sabelli and L. Kovacevic, "Quantum bios and biotic complexity in the distribution of galaxies". Complexity, vol. 11, no. 1, pp. 14 25, 2006.

[13] G. Thomas, H. Sabelli, L. Kovacevic, and L. Kauffman, "Biotic patterns in Schrödinger's equation and the evolution of the universe", Inter. J. Complex Sys., electronic publication manuscript number $1787,2006$. 
[14] H. Sabelli and L. Kovacevic, "Biotic Expansion of the Universe", International Conference on Advances in Internet, Processing, Systems, and Interdisciplinary Research, electronic publication, 2003.

[15] H. Sabelli, L. Carlson-Sabelli, M. Patel, J. Zbilut, J. Messer, and K. Walthall, "Psychocardiological portraits: A clinical application of process theory", Chaos theory in Psychology. Eds. F. D. Abraham and A. R. Gilgen, Greenwood Publishing Group Inc., Westport, CT, 1995.

[16] H. Sabelli and L. Kovacevic, "Biotic population dynamics", Complexity, (In press).

[17] H. Sabelli, "Bios Theory of Physical, Biological and Human Evolution", Explorations in Complexity Thinking. Eds. K. A. Richardson and P. Cilliers, ISCE Publishing, Mansfield, MA, 2007.

[18] H. Sabelli, "Bios, creative organization in economic, biological, and meteorological data", International Conference on Advances in Internet, Processing, Systems, and Interdisciplinary Research, electronic publication, 2003.

[19] A. Levy, D. Alden, C. Levy, "Biotic Patterns in Music", Society for Chaos Theory in Psychology and Life Sciences Conference, 2006.

[20] L. Kauffman and H. Sabelli, "The Process equation", Cybern. Sys., vol. 29, no. 4, pp. 345-362, June 1998.

[21] H. Sabelli, Bipolar Feedback, Encyclopedia of Life Support Systems, Eolss Publishers, Oxford, UK, 2003.

[22] E. J. Chaisson, Cosmic Evolution, Harvard University Press, Cambridge, MA, 2001

[23] E. Schrödinger, "What is Life?", Cambridge University Press, Cambridge, UK, 1945.

[24] I. Prigogine, From Being to Becoming. W H Freeman, San Francisco, CA, 1980.

[25] G. Bateson, "Steps to An Ecology of Mind: Collected Essays in Anthropology, Psychiatry, Evolution, and Epistemology", Chandler Publishing Company, San Francisco, CA, 1972.

[26] J. B. S. Haldane, "Pasteur and cosmic asymmetry", Nature, vol. 185 , no. 1 , pp. 87 , January 1960.

[27] G. Spencer-Brown, Laws of Form, E. P. Dutton, New York, NY, 1979.

[28] L. Carlson-Sabelli, H. Sabelli, M. Patel, and K. Holm, "The union of opposites in sociometry: an empirical application of process theory", J. Group Psychother. Psychodrama. Sociom., vol. 44, no. 4, pp. 147-171, 1992.

[29] L. H. Kauffman, "Pendulum Traced Curves and Damped Oscillations in the Plane", J. Knot Theor. Ramif., vol. 1, no. 1, pp. 21, 2007.

[30] H. Sabelli, "Complement plots: analyzing opposites” Int. J. General Syst., vol. 29, no. 5, pp. 799-830, 2000.

[31] J. L. O’Brien, "Optical Quantum Computing”, Science, vol. 318, no. 1 , pp. $1567-1570,2007$.
[32] H. Sabelli and G. Thomas. Eds., F. Orsucci and N. Sala, The Future Quantum Computer: Biotic Complexity, Reflecting Interfaces: The Complex Coevolution of Information Technology Ecosystems, Idea Group, Hershey, (In press).

[33] G. J. Chaitin, "Information, Randomness and Incompleteness", World Scientific, Toh Tuck Link, Singapore, 1987.

[34] N. Bourbaki, "L'architecture des mathématiques", Les grands courants de la pensée mathématique. Ed. F. Le Lionnais, Cahiers du Sud, Paris, France, 1948.

[35] E. W. Beth and J. Piaget, Épistémologie mathématique et psychologie, Presses Universitaires de France, Paris, France, 1961.

[36] H. O. Peitgen, H. Jürgens, and D. Saupe, Chaos and Fractals. Springer, New York, NY, 1992.

[37] I. Lakatos, Proofs and Refutations, Eds. J. Worrall and E. Zahar, Cambridge University Press, Cambridge, UK, 1976.

[38] C. E. Shannon and W. Weaver, The Mathematical Theory of Communication, The University of Illinois Press, Urbana, IL, 1964.

[39] H. Sabelli, "Mathematical Development: A theory of natural creation", Kybernetes, vol. 32, no. 5/6, pp. 752-766, 2003.

[40] H. Sabelli and L. Carlson-Sabelli, "Biological priority and psychological supremacy: a new integrative paradigm derived from process theory", Am. J. Psychiatry, vol. 146, no. 12, pp. 1541-1551, December 1989.

[41] J. G. Miller, Living Systems. McGraw-Hill, New York, NY, 1978.

[42] N. Winner, "Cybernetics, or Control and Communication in the Animal and the Machine", MIT Press, Cambridge, MA, 1948.

[43] K. F. Riegel, "Foundations of Dialectical Psychology", Academic Press Inc., New York, NY, 1979.

[44] M. D. Gumer, "Payment for sex in a macaque mating market", Anim. Behav., vol. 74, no. 2, pp. 1655-1667, 2007.

[45] C. A. Torre, "Chaos in the Triadic Theory of Psychological Competence in the Academic Setting", Chaos Theory in Psychology. Eds. F. D. Abraham and A. R. Gilgen, Greenwood Publishing Group Inc., Westport, CT, 1995.

[46] P. Wheelwright, Heraclit. Princeton University Press, Princeton, NJ, 1959.

[47] F. Engels, "Dialectics of Nature", International Publishers, New York, NY, 1960.

[48] G. Boole, The Laws of Thought, Dover Publications, New York, NY, 1854.

[49] M. Kosok, "The Dynamics of Hegelian Dialectics, and Nonlinearity in the Sciences, Hegel and the Sciences", Eds. R. S. Cohen and M. W. Wartofsky, D. Reidel Publishing Co., New York, NY, 1984.

[50] H. Sabelli, "Mathematical Dialectics, Scientific Logic and the Psychoanalysis of Thinking, Hegel and the Sciences", Eds. R. S. Cohen and M. W. Wartofsky, D. Reidel Publishing Co., New York, NY, 1984. 